\title{
Treatment of the antiphospholipid syndrome
}

\author{
Monica Galli
}

Received: 21 November 2013/Accepted: 26 November 2013/Published online: 22 December 2013

(C) Springer-Verlag Italia 2013

\begin{abstract}
The antiphospholipid syndrome is characterized by a combination of laboratory findings (i.e., the presence of at least one antiphospholipid antibody) and clinical manifestations (arterial and/or venous thrombosis, obstetrical complications). Long-term oral anticoagulant is recommended to prevent recurrence of both arterial and venous thrombosis, whereas (low molecular weight) heparin plus aspirin is the treatment of choice to prevent further obstetrical complications. In the rare case of catastrophic antiphospholipid syndrome, heparin plus highdose corticosteroids plus plasma exchange is associated with the highest recovery rate. Some new, non-antithrombotic-based treatments of antiphospholipid syndrome with rituximab, autologous stem cell transplantation, or hydroxychloroquine are also reviewed.
\end{abstract}

Keywords Antiphospholipid antibodies .

Antiphospholipid syndrome · Thrombosis · Pregnancy loss

\section{Introduction}

The history of antiphospholipid (aPL) antibodies started almost 25 years ago, when the first reports of $\beta 2$-glycoprotein I ( $\beta 2$-GPI) [1-3] and prothrombin [4] as antigenic targets of these antibodies were published. During more than two decades, knowledge has accumulated that clarified the interconnection between lupus anticoagulant (LA) activity measured in phospholipid-dependent coagulation

M. Galli ( ()

UO Ematologia, Ospedale Papa Giovanni XXIII, Largo OMS, 1, 24127 Bergamo, Italy

e-mail: monicagalli@hpg23.it tests and antibodies directed against cardiolipin (aCL), $\beta 2$ GPI (antiß2-GPI), and prothrombin measured by enzymelinked immunosorbent assays (ELISA) [1, 4]. It is now well accepted that LA activity may be exerted via different mechanisms by both antiprothrombin [4] and antiß2-GPI antibodies [5], either alone or in combination [5, 6], and that the majority of aCL antibodies recognize, in reality, $\beta 2$-GPI bound to cardiolipin-coated plate [1]. These observations guided the establishment of the clinical and laboratory criteria for antiphospholipid syndrome (APS) first in 1999 [7], which were updated in 2006 [8]. In 2009 the updated laboratory criteria for LA diagnosis, first set in 1985 [9], were published [10].

\section{Pathophysiology of APS}

That aPL antibodies are causatively related to the clinical manifestations of APS and not merely innocent markers of these events comes from a large body of studies, which demonstrated that:

1. the infusion of plasma or the total $\operatorname{IgG}$ fraction or the affinity-purified aPL antibodies from patients with APS increases the thrombus size in ex vivo models of arterial and venous thrombosis in mice and hamsters,

2. passive and active immunization with total $\operatorname{IgG}$ fraction or affinity-purified aPL antibodies from patients with APS increases the fetal resorption rate of pregnant mice and causes thrombocytopenia,

3. myocardial infarction, thrombocytopenia and increased fetal resorption rate are typical of mice prone to systemic lupus erythematosus (SLE).

A comprehensive review on this topic has been recently published [11]. 
From a mechanistic point of view, particular focus has been paid to the prothrombotic effects of antiß2-GPI antibodies, as their interaction with $\beta 2$-GPI occurs on a phospholipid surface. This may lead to a prothrombotic condition. In this respect, impairment of factor $\mathrm{Va}$ inactivation by the protein $\mathrm{C}$ system [12], displacement of annexin $\mathrm{V}$ from anionic procoagulant surfaces [13] and suppression of the inhibitory activity of tissue factor pathway inhibitor [14] have been described to be caused by anti $\beta 2-$ GPI antibodies in the presence of $\beta 2-$ GPI. Also, antiprothrombin antibodies have been shown to inhibit factor $\mathrm{Va}$ inactivation by the protein $\mathrm{C}$ system in a prothrombin-dependent way [15]. This is, however, a too simplistic explanation of the prothrombotic effect of antiß2-GPI antibodies. In their recent review, Giannakopoulos and Krilis [16] have reported a long list of other possible or ascertained mechanisms through which anti $\beta 2$ GPI antibodies may lead to thrombosis: increased oxidative stress, impaired function of endothelial nitric oxide synthase, activation of receptors, increased expression and activation of tissue factor, increase in free thiol form of Factor XI, antibody-mediated activation of complement C3 and C5, increased expression of Toll-like receptors 7 and 8 [17], increased sensitization of Toll-like receptors 7 and 8 agonists and $\mathrm{B}$ cell activating factor.

Among anti $\beta 2-G P I$ antibodies, those directed against the Gly40-Arg43 peptide sequence on domain I express LA activity and are, obviously, also positive in ELISAs for aCL and antiß2-GPI antibodies [18, 19]. Under normal circumstances, the Gly40-Arg43 peptide epitope on domain $\mathrm{I}$ is hidden and cannot, therefore, elicit binding of antiß2-GPI antibodies. However, upon binding to an anionic phospholipid surface via the large positive patch on domain V, $\beta 2$-GPI undergoes a conformational change which uncovers the hidden epitope [20]. Such a binding may occur on the surface of an injured vessel or on the membrane of activated blood or endothelial cells or platelets. This is coherent with the "two-hits" hypothesis, which explains why aPL antibodies are not thrombogenic per se.

$\beta 2-$ GPI may circulate in plasma in both a circular and an open conformation form [21]. The circular form is maintained by the interaction between the first and the fifth domain and is non-immunogenic; conversely, the open form exposes the Gly40-Arg43 peptide epitope, which becomes available for the binding of the thrombogenic antiß2GPI antibody. Under oxidative stress conditions, the balance between these two forms may shift toward the open conformation of $\beta 2-$ GPI. In this respect, Ioannou et al. [22] have recently reported elevated levels of oxidized $\beta 2 \mathrm{GPI}$ in APS patients. In its oxidized form, $\beta 2 \mathrm{GPI}$ is unable to protect endothelial cells against oxidative stress-induced cell injury [23]. Therefore, it is conceivable that the relative abundance of oxidized $\beta 2$ GPI in APS may lower the threshold for development of vascular thrombosis by itself as well as via its interaction with antiß2GPI antibodies.

The ontogeny of the pathogenic aPL antibodies is still poorly understood. According to the "molecular mimicry" theory, the incidental exposure to certain infectious agents that bear $\beta 2 \mathrm{GPI}$-like structures may induce a break in tolerance to this antigen and facilitate the production of pathogenic aPL in genetically prone subjects [24]. Infectious and/or inflammation agents or oxidative stress may also induce conformational changes in $\beta 2 \mathrm{GPI}$, with the consequent exposure of otherwise hidden epitopes, which enables autoantibody production [25].

\section{APS manifestations and risk profile}

The analysis of clinical events at disease onset of 1,000 APS patients showed the following prevalences: deep vein thrombosis $31.7 \%$, cerebral stroke $13.1 \%$, superficial thrombophlebitis $9.1 \%$, pulmonary embolism $9 \%$, transient ischemic attacks $7 \%$, myocardial infarction $2.8 \%$ and fetal losses $8.5 \%$ [26]. The 5-year follow-up analysis of the same cohort of patients showed that 200 patients $(20 \%)$ developed APS-related manifestations: of them, 166 were recurrent thrombosis [27]. The most common were strokes, transient ischemic attacks, deep vein thrombosis and pulmonary embolism (in decreasing order of prevalence).

Systematic reviews have consistently reported that LA is a stronger risk factor than aCL and antiß2-GPI for both arterial and venous thrombosis and obstetric complications [28-30]. aCL and antiß2-GPI antibodies only show some significant association with thrombosis and obstetric complications at high titer. In keeping with this is the observation that when they are independent of LA and aCL, neither antiß2-GPI nor antiprothrombin antibodies are associated with arterial or venous thrombosis [31].

Moving from the prognostic significance of the single aPL test to that of the aPL profile-defined by the various combinations of LA, aCL and antiß2-GPI positivity [32]several retrospective and prospective studies have shown that triple aPL positivity (i.e., aCL, antiß2-GPI and LA positivity) correlates more strongly with both thrombosis and pregnancy morbidity than the presence of single or double positivity [32-39]. With respect to thrombosis, triple aPL positivity conferred an odds ratio (OR) that ranged from 5.24 to 33.3. In contrast, none of the other combinations of aPL positivities reached statistical significance. A large, prospective multicenter study on triple aPL-positive APS patients reported a cumulative incidence of thrombosis of 12.2, 26.1 and $44.2 \%$ after 1,5 and 10 years of follow-up, respectively [34]. 
Antibodies responsible for triple aPL positivity recognize the Gly40-Arg43 epitope in the first domain of $\beta 2$-GPI and are associated with a history of thrombosis $[18,19]$. When measured by means of an ELISA with the first domain of $\beta 2$-GPI coated on hydrophobic plates, the prevalence of $\operatorname{IgG}$ antibodies was $55 \%$ among $442 \beta 2$ GPI-positive patients [39]. Their presence conferred an OR of 3.5 and 2.4 for thrombosis and obstetric complications, respectively.

\section{Primary prevention of thrombosis}

The appropriateness of primary thromboprophylaxis in asymptomatic aPL-positive subjects is still under debate. A trial on 98 such patients who were randomized to receive either aspirin or placebo failed to show any benefit from aspirin administration [40]. Furthermore, the overall annual incidence of first acute thrombosis was very low in both groups of patients $(2.7 \%$ patient-year for aspirin-treated patients and $0 \%$ patient-year for those receiving placebo). These figures correlate well with the observation from the Italian Registry on aPL antibodies, which reported an incidence rate of first thrombosis of $0.95 \%$ patient-year among 243 aPL-positive cases without a history of thrombosis [41]. These data, however, do not take into account the aPL profiles of the patients.

The group of Pengo et al. [35] has reported a clear correlation between the risk of first thrombosis and the aPL profile: compared to an annual rate of $0.4 \%$ in the normal white population aged 35-55 years, that of single aPLpositive asymptomatic patients carries approximately $1.36 \%$ [42], and that of triple aPL-positive asymptomatic patients exceeds $5 \%$. The cumulative incidence of first thrombotic event among their 104 triple aPL-positive patients was $9.8 \%$ after 2 years, $27.3 \%$ after 5 years and $37.1 \%$ after 10 years. There were 12 arterial and 11 venous TEs, 1 thrombosis of the right atrium and 1 renal thrombotic microangiopathy. Most carriers of triple positivity in the study $(64 \%)$ did not receive any antithrombotic prophylaxis and the remaining were given low-dose aspirin. However, aspirin did not reduce the rate of either total or arterial thrombosis.

Very recently, the results of a trial in 166 aPL-positive patients with SLE and/or obstetric morbidity randomized to receive either low-dose aspirin $(n=82)$ or low-dose aspirin plus low-intensity warfarin $(n=84)$ have been reported [43]. No difference in the number of thrombosis between the two groups was observed (four events in each group) and the overall incidence of thrombosis was $1.8 \%$ patients-year. More episodes of hemorrhage were recorded in the group receiving low-dose aspirin plus low-intensity warfarin. The study included also an observational arm of
66 patients, who had declined randomization, 65 of whom were on low-dose aspirin. Their incidence of thrombosis was $4.9 \%$ patients-year (7 events in 66 patients). This is a rather unexpected result, particularly when compared with the outcome of the group of patients randomized to lowdose aspirin.

Based on these data, one may consider primary thromboprophylaxis only in triple aPL-positive asymptomatic subjects, or in those carrying an underlying (autoimmune) disease. It is, however, necessary to remind that aspirin is not the drug of choice. Whether these patients should receive oral anticoagulation (OAT) (either vitamin $\mathrm{K}$ antagonists or one of the new oral anticoagulants) or drugs that target one or more among the possible pathogenic mechanisms of thrombosis still has to be demonstrated. Of course, strict control and, whenever possible, treatment of venous and arterial risk factors and adequate thromboprophylaxis during high-risk situations are of the utmost importance to reduce the risk of the first thrombotic event.

\section{Secondary prevention of recurrent thrombosis}

Long-term oral OAT with vitamin $\mathrm{K}$ antagonists is the mainstay of treatment of APS patients to prevent the recurrence of both arterial and venous thrombosis. In general, a low-intensity OAT (aimed at a PT INR between 2.0 and 3.0) is recommended after the first episode of venous thrombosis. After an arterial event, OAT at higher intensity (aimed at a PT INR 3.0-4.0) or a combination therapy is recommended. In the case of a second recurrence despite an adequate OAT (at a PT INR 2.0-3.0), it is suggested to increase its intensity to reach a PT INR $3.0-4.0$ or to shift to alternative therapies. Patients with aPL antibodies at low titer or with a single aPL positivity should receive secondary treatment as per usual recommendations. For recent reviews see references [44-47]. Evidence-based recommendations on prevention and treatment of thrombosis have been published recently [47].

These recommendations take into account, at least partially, the results of several retrospective studies and of three randomized clinical trials (RCT). Two RCTs [48, 49] specifically addressed the issue of the optimal intensity of OAT and concluded that a low intensity (aimed at a PT INR 2.0-3.0) was equally efficient as a higher intensity regimen (aimed at a PT INR 3.0-4.0). However, they were limited by the relatively small number of patients included in each study and by the enrollment of patients with both arterial and venous thrombosis as the qualifying event. The third RCT [50] addressed the issue of the prevention of thrombosis recurrence after a first stroke and concluded that aspirin ( $325 \mathrm{mg} /$ day) was equally efficient and safe as OAT (aimed at a PT INR 1.4-2.8). However, this was a 
sub-study of a larger study, the presence of aPL antibodies was measured only once and most cases were at low aPL titer. Thus, the majority of the patients included in the trial did not qualify for a diagnosis of definite APS.

The overall incidence of recurrent thrombosis in APS patients approximates 4-6\% patients-year [34, 41]. Patients off OAT have a significantly higher risk to develop a recurrence: in the study by Pengo et al. [34] on a large number of well-characterized triple-positive APS patients, thrombosis recurred in $29 \%$ of those on OAT and in $51 \%$ of those not receiving OAT. Thus, this study supports the concept of a prolonged duration of secondary prophylaxis. However, the same study reported that the incidence of thrombosis recurrence was highest in the first year $(12.2 \%)$ and declined thereafter. On the other side, it clearly shows that high-risk (i.e., triple aPL positive) patients may not be sufficiently protected by OAT.

Thus, when approaching the issue of prevention of recurrent thrombosis in APS patients, a number of key questions still need a proper answer:

1. Should arterial and venous thrombosis be treated differently?

2. Which is the optimal intensity of anticoagulation?

3. Which is the optimal duration of anticoagulation?

4. Should APS patients receive different treatment according to their aPL risk profile?

5. What is the role of the new oral anticoagulant drugs in APS?

6. Should non-antithrombotic drugs be considered in the secondary prevention of thrombosis?

No data are, so far, available regarding the safety and efficacy of new oral anticoagulant drugs in APS patients. It is possible that such information will be available in the coming years. In fact, the prospective phase II/III randomized RAPS (Rivaroxaban in APS) trial is an Arthritis Research UK study presently underway, aimed at demonstrating that the intensity of anticoagulation in thrombotic APS patients on rivaroxaban is non-inferior to that obtained with warfarin as assessed by the endogenous thrombin potential.

Regarding the non-antithrombotic drug approach of APS, a number of small studies and case reports have reported about the outcome of patients treated with rituximab or autologous stem cell transplantation (ASCT). Rituximab has been used mainly to treat non-thrombotic manifestations of APS: a recent phase II trial showed a complete or partial response in terms of thrombocytopenia, skin ulcers, nephropathy and cognitive dysfunctions in 11 out of 19 patients [51]. No amelioration of cardiac valve disease was seen. aPL titers did not change over the 1-year follow-up period. ASCT is feasible and safe in APS patients and may eradicate aPL antibodies, at least in a proportion of patients [52]. A trial of ASCT in 22 SLE patients with APS showed that 18 of them were able to discontinue OAT, with $78 \%$ remaining thrombosis free after a median follow-up of 15 months [52]. No ASCTrelated mortality was reported.

The so-called "seronegative" APS, patients whose aPL antibodies turn negative, and aPL-positive patients who do not formally satisfy the criteria for APS represent unusual situations [53] which pose particularly challenging treatment problems [54].

\section{Primary and secondary prevention of obstetric complications}

Approximately, 15-20\% of women with three or more pregnancy losses are aPL positive [55], and their reported fetal loss rate reaches $50-90 \%$ without specific treatments [56]. Thus, in the last 20 years a number of clinical trials have been performed to identify the most appropriate treatment to improve the pregnancy outcome of these women.

Primary prevention of obstetric complications in asymptomatic aPL-positive women is generally managed with patient's education and strict controls of pregnancy. Administration of low-dose aspirin, particularly in the case of a concomitant autoimmune disease, may be considered [57].

In thrombotic APS women who want to undergo their first pregnancy, treatment is mostly aimed at preventing thrombosis recurrence [58]. Therefore, they are normally treated with full-dose low molecular weight heparin (LMWH) with or without aspirin throughout pregnancy. OAT is generally resumed at puerperium. In case the thrombotic APS patient is on aspirin prophylaxis before pregnancy, aspirin should be continued and LMWH addition (at full or prophylactic dose) considered during pregnancy. In case the thrombotic APS patient is not receiving any antithrombotic drug, administration of LMWH at least at prophylactic dose should be considered throughout pregnancy. These approaches favor also the positive outcome of pregnancy, since thrombotic APS women appear to have a higher risk of preterm delivery and small for gestational age babies compared to obstetric APS [59].

Secondary prevention of obstetric complications is aimed at improving both maternal and neonatal outcomes and is based on the administration of LMWH at prophylactic dose plus low-dose aspirin [44]. This recommendation derives from a number of clinical trials performed during the last 20 years, which also demonstrated that prednisone or intravenous immunoglobulins were not beneficial and may be even detrimental to pregnancy outcomes. These trials, however, did not solve the problem of 
the best timing for commencing both aspirin and LMWH. Again, a distinction should be made according to the thrombotic or obstetric APS phenotype of these women. In purely obstetric APS women, pregnancy may be successfully managed according to protocols based on LMWH and aspirin [59]. Conversely, history of both thrombosis and pregnancy loss confers to APS women an odds ratio of unsuccessful pregnancy of 12.7 despite adequate treatment [59]. Also, the presence of SLE or other autoimmune diseases was an independent risk factor of adverse outcomes [59]. When analyzed according to their aPL profile, triple aPL-positive women have the highest risk of pregnancy losses [33, 60, 61]. Thus, more studies that stratify patients according to their clinical phenotype and aPL profiles are urgently needed to establish the most appropriate treatment strategies for these women at high risk of unsuccessful pregnancy despite conventional therapy.

\section{The catastrophic APS (CAPS)}

Catastrophic APS (CAPS) is a rare variant of APS occurring in $<1 \%$ of the patients. It is characterized by thrombosis in multiple organs over a short period of time, with histopathological evidence of occlusions of small vessels [62]. Since 2000, an International Registry has been created [63] aimed at documenting the clinical and laboratory features of these cases, the treatment strategies employed and the outcome of treatments.

The pathogenesis of CAPS is still poorly understood. (Bacterial) infections anticipate its development in approximately $60 \%$ of the patients [64]. The clinical manifestations of CAPS depend on the organs affected by the thrombotic events, extent of thrombosis and of systemic inflammatory response syndrome. Cerebral involvement (due to stroke, cerebral hemorrhage or encephalopathy) is the main cause of death, followed by cardiac involvement and infection. Coexistent SLE is the only recognized adverse prognostic factor of a higher mortality rate, which, before 2000, exceeded $50 \%$ and went down to about 20-30\% in the last decade [65].

First-line treatment of CAPS includes heparin plus highdose corticosteroids plus plasma exchange, which is associated with the highest recovery rate $(78 \%)$, followed by heparin plus high-dose corticosteroids plus infusion of immunoglobulins (69\% recovery rate) [65]. An algorithm for treatment of CAPS has been proposed already a decade ago [66]. A follow-up study of CAPS survivors showed that about $17 \%$ of them developed further APS-related manifestations, but none of them relapsed of CAPS [67]. The registry, however, reports about few patients who relapsed of CAPS: infections, sub-therapeutic OAT or withdrawal of OAT were the most probable precipitating factors.

A recent analysis from the "CAPS Registry" reported on 20 patients treated with rituximab: 8 of them $(40 \%)$ received rituximab in combination with the first-line therapy (in 2 cases as part of lymphoma treatment), whereas the other $12(60 \%)$ as second-line therapy [68]. The dosage and scheme of rituximab administration were rather variable. Overall, 16 patients $(80 \%)$ recovered from CAPS, whereas the other $4(20 \%)$ died.

\section{Conclusions and future trends in the treatment of APS}

The identification of the antigenic targets of aPL antibodies allowed a new start in the investigation of the mechanisms of thrombosis and pregnancy loss that characterize APS. It is now ascertained that antiß2-GPI antibodies toward the Gly40-Arg43 epitope in the first domain of $\beta$-GPI play an important pathogenic role, whose intracellular pathways are being clarified. This is allowing physicians to consider new, not necessarily anticoagulation-based, therapeutic approaches of APS. Besides Rituximab and ASCT, other drugs have already been employed in APS. For instance, hydroxychloroquine-treated SLE patients have been recently reported to have reduced odds to display a persistent aPL positivity [69, 70]. Another study [71] showed that 1-month administration of fluvastatin to 42 APS patients significantly reduced their expression of monocyte tissue factor, protein activator receptors 1 and 2 and vascular endothelial growth factor. Moreover, proteins involved in thrombotic development (annexin II, RhoA and protein disulfide isomerase) displayed an altered expression after fluvastatin administration. Presently, several molecules targeting different pathways of cell activation due to aPL or antiß2-GPI antibodies are being tested in various in vitro and ex vivo models of APS. Thus, the possibility that immunomodulatory drugs and targeted therapies will efficiently treat or, even better, prevent the clinical manifestations of APS does not seem anymore too far away from being realized. This will translate into a significant improvement of the quality and also the quantity of the life of patients suffering from APS.

Conflict of interest The author states that she has no conflicts of interest.

Human and animal rights The contents of the manuscript do not violate human and animal rights.

Informed consent The contents of the manuscript do not require an informed consent. 


\section{References}

1. Galli M, Comfurius P, Maassen C et al (1990) Anticardiolipin antibodies (ACA) directed not to cardiolipin but to a plasma protein cofactor. Lancet 334:1544-1547

2. McNeil HP, Simpson RJ, Chesterman CN, Krilis SA (1990) Antiphospholipid antibodies are directed against a complex antigen that includes a lipid-binding inhibitor of coagulation: $\beta 2$-glycoprotein I (apolipoprotein H). Proc Natl Acad Sci USA 87: 4120-4124

3. Matsuura E, Igarashi Y, Fujimoto M et al (1990) Anticardiolipin cofactor(s) and differential diagnosis of autoimmune disease. Lancet 336:177-178

4. Bevers EM, Galli M, Barbui T, Comfurius P, Zwaal RFA (1991) Lupus anticoagulant IgG's (LA) are not directed to phospholipids only, but to a complex of lipid-bound human prothrombin. Thromb Haemost 66:629-632

5. Galli M, Comfurius P, Barbui T, Zwaal RFA, Bevers EM (1992) Anticoagulant activity of anti- $\beta 2$-glycoprotein I is potentiated by a distinct subgroup of anti-cardiolipin antibodies. Thromb Haemost 68:297-300

6. Horbach DA, van Oort E, Derksen RHWM, de Groot PG (1998) The contribution of anti-prothrombin antibodies to lupus anticoagulant activity. Thromb Haemost 9:790-796

7. Wilson WA, Gharavi AE, Koike T et al (1999) International consensus statement on preliminary classification criteria for definite antiphospholipid syndrome. Arthr Rheum 42:1309-1311

8. Miyakis S, Lockshin MD, Atsumi T et al (2006) International consensus statement on an update of the classification criteria for definite antiphospholipid syndrome (APS). J Thromb Haemost 4:295-306

9. Brandt JT, Triplett DA, Alving B, Scharrer I (1995) Criteria for the diagnosis of lupus anticoagulants: an update. On behalf of the Subcommittee on Lupus Anticoagulant/Antiphospholipid Antibody of the Scientific and Standardization Committee of the ISTH. Thromb Haemos 74:1185-1190

10. Pengo V, Tripodi A, Reber G et al (2009) Update of the guidelines for lupus anticoagulant detection. J Thromb Haemost 7:1737-1740

11. Willis R, Pierangeli S (2011) Pathophysiology of the antiphospholipid syndrome. Autoimmun Highlights 2:35-52

12. Galli M, Ruggeri M, Barbui T (1998) Differential effects of antiß2-Glycoprotein I and anti-prothrombin antibodies on the anticoagulant activity of activated protein C. Blood 91: 1999-2004

13. Rand JH, Wu XX, Quinn AS, Taatjes DJ (2010) The annexin A5mediated pathogenic mechanism in the antiphospholipid syndrome: role in pregnancy losses and thrombosis. Lupus 19:460-469

14. Salemink I, Blezer R, Willems GM et al (2000) Antibodies to beta2-glycoprotein I associated with antiphospholipid syndrome suppress the inhibitory activity of tissue factor pathway inhibitor. Thromb Haemost 84:653-656

15. Galli M, Willems GW, Rosing J et al (2005) Anti-prothrombin IgG from patients with anti-phospholipid antibodies inhibits the inactivation of factor $\mathrm{Va}$ by activated protein $\mathrm{C}$. Br J Haematol 129:240-247

16. Giannakopoulos B, Krilis SA (2013) The pathogenesis of the antiphospholipid syndrome. N Engl J Med 368:1033-1048

17. Von Landberg P (2011) Toll-like receptors play a crucial part in the pathophysiological activity of antiphospholipid antibodies. Autoimmun Highlights 2:53-57

18. de Laat BH, Derksen RHWM, Urbanus R, Roest M, de Groot PG (2004) $32-G P I-d e p e n d e n t ~ l u p u s$ anticoagulant activity highly correlates with thrombosis in the antiphospholipid syndrome. Blood 104:3598-3602
19. de Laat BH, Derksen RHWM, Urbanus R, Roest M, de Groot PG (2005) IgG antibodies that recognize epitope Gly40-Arg43 in domain I of $\beta 2$-glycoprotein I cause LAC, and their presence correlates strongly with thrombosis. Blood 105:1540-1545

20. de Laat BH, Derksen RHWM, Van Lummel M, Pennings MT, de Groot PG (2006) Pathogenic anti- $\beta 2$-glycoprotein I antibodies recognize domain I of $\beta 2$-glycoprotein I only after a conformational change. Blood 107:1916-1924

21. Agar C, Van Os GM, Morgelin M et al (2010) Beta2-glycoprotein I can exist in 2 conformations: implications for our understanding of the antiphospholipid syndrome. Blood 116:1336-1343

22. Ioannou I, Zhang YJ, Qi M et al (2011) Novel Assays of thrombogenic pathogenicity in the Antiphospholipid Syndrome based on the detection of molecular oxidative modification of the major autoantigen 32 -Glycoprotein I. Arthr Rheum 63:2774-2782

23. Ioannou Y, Zhang JY, Passam FH et al (2010) Naturally occurring free thiols within $\beta 2$-glycoprotein I in vivo: nitrosylation, redox modification by endothelial cells and regulation of oxidative stress induced cell injury. Blood 116:1961-1970

24. Gharavi AE, Pierangeli SS, Harris EN (2001) Origin of the antiphospholipid antibodies. Rheum Dis Clin North Am 27:551-563

25. Van OSGM, Meijers JC, Agar C et al (2011) Induction of anti$\beta 2$-glycoprotein I autoantibodies in mice by protein H of Streptococcus pyogenes. J Thromb Haemost 9:2447-2456

26. Cervera R, Piette JC, Font J et al (2002) Antiphospholipid syndrome: clinical and immunologic manifestations and patterns of disease expression in a cohort of 1,000 patients. Arthr Rheum 46:1019-1027

27. Cervera R, Khamashta MA, Shoenfeld Y et al (2009) Morbidity and mortality in the antiphospholipid syndrome during a 5-year period: a multicentre prospective study of 1,000 patients. Ann Rheum Dis 68:1428-1432

28. Galli M, Luciani D, Bertolini G, Barbui T (2003) Lupus anticoagulants are stronger risk factors of thrombosis than anticardiolipin antibodies in the antiphospholipid syndrome: a systematic review of the literature. Blood 101:1827-1832

29. Galli M, Luciani D, Bertolini G, Barbui T (2003) Antiß2-glycoprotein I, antiprothrombin antibodies and the risk of thrombosis in the antiphospholipid syndrome. Blood 102:2717-2723

30. Opatrny L, David M, Kahn SR, Shrier I, Rey E (2006) Association between antiphospholipid antibodies and recurrent fetal loss in women without autoimmune diseases: a metaanalysis. J Rheumatol 33:2214-2221

31. Previtali S, Barbui T, Galli M (2002) Anti- $\beta 2$-glycoprotein I and antiprothrombin antibodies in antiphospholipid-negative patients with thrombosis: a case-control study. Thromb Haemost 88:729-732

32. Pengo V, Biasiolo A, Pegoraro C, Cucchini U, Noventa F, Iliceto S (2005) Antibody profiles for the diagnosis of antiphospholipid syndrome. Thromb Haemost 93:1147-1152

33. Ruffatti A, Tonello M, Del Ross T et al (2006) Antibody profile and clinical course in primary antiphospholipid syndrome with pregnancy morbidity. Thromb Haemost 96:337-341

34. Pengo V, Ruffatti A, Legnani C et al (2010) Clinical course of high-risk patients diagnosed with antiphospholipid syndrome. J Thromb Haemost 8:237-242

35. Pengo V, Ruffatti A, Legnani $C$ et al (2011) Incidence of a first thromboembolic event in asymptomatic carriers of high risk antiphospholipid antibody profile: a multicentre prospective study. Blood 118:4714-4718

36. Lee EY, Lee CK, Lee TH et al (2003) Does the anti- $\beta 2$-glycoprotein I antibody provide additional information in patients with thrombosis? Thromb Res 111:29-32 
37. Male C, Foulon D, Hogendoorn $\mathrm{H}$ et al (2005) Predictive values of persistent versus transient antiphospholipid antibody subtypes for the risk of thrombotic events in pediatric patients with systemic lupus erythematosus. Blood 106:4152-4158

38. Forastiero R, Martinuzzo M, Pombo G et al (2005) A prospective study of antibodies to $\beta 2$-GPI and prothrombin and risk of thrombosis. J Thromb Haemost 3:1231-1238

39. De Laat B, Pengo V, Pabinger I et al (2009) The association between circulating antibodies against domain I of beta2-glycoprotein I and thrombosis: an international multicenter study. J Thromb Haemost 7:1767-1773

40. Erkan D, Harrison MJ, Levy M et al (2007) Aspirin for primary thrombosis prevention in the antiphospholipid syndrome: a randomized, double-blind, placebo-controlled trial in asymptomatic antiphospholipid antibody-positive individuals. Arthr Rheum 56:2882-2891

41. Finazzi G, Brancaccio V, Moia M et al (1996) Natural history and risk factors for thrombosis in 360 patients with antiphospholipid antibodies: a four-year prospective study from the Italian Registry. Am J Med 100:530-536

42. Ruffatti A, Ross TD, Ciprian M et al (2011) Risk factors for a first thrombotic event in antiphospholipid antibody carriers: a prospective multicentre follow-up study. Ann Rheum Dis 70:1083-1086

43. Cuadrado MJ, Bertolaccini ML, Seed PT (2013) Low-dose aspirin vs low-dose aspirin plus low-intensity warfarin in thromboprophylaxis: a prospective, multicentre, randomized, open, controlled trial in patients positive for antiphospholipid antibodies (ALIWAPAS). Rheumatology E-pub October 4

44. Ruiz-Irastorza G, Crowther M, Branch W, Khamashta MA (2010) Antiphospholipid syndrome. Lancet 376(1498-150):9

45. Derksen RHWM, de Groot PG (2010) Towards evidence-based treatment of thrombotic antiphospholipid syndrome. Lupus $19: 470-474$

46. Punnialingam S, Khamashta MA (2013) Duration of anticoagulation treatment for thrombosis in APS: is it ever safe to stop? Curr Rheumatol Rep 15:318-325

47. Ruiz-Irastorza G, Cuadrado MJ, Ruiz-Arruza I et al (2011) Evidence-based recommendations for the prevention and long-term management of thrombosis in antiphospholipid antibody-positive patients: report of a task force at the 13th International Congress on antiphospholipid antibodies. Lupus 20:206-218

48. Crowther MA, Ginsberg JS, Julian J et al (2003) A comparison of two intensities of warfarin for the prevention of recurrent thrombosis in patients with the antiphospholipid syndrome. N Engl J Med 349:1133-1138

49. Finazzi G, Marchioli R, Brancaccio V et al (2005) A randomized clinical trial of high-intensity warfarin vs. conventional antithrombotic therapy for the prevention of recurrent thrombosis in patients with the antiphospholipid syndrome (WAPS). J Thromb Haemost 3:848-853

50. Levine SR, Brey RL, Tilley BC, APASS Investigators et al (2004) Antiphospholipid antibodies and subsequent thromboocclusive events in patients with ischemic stroke. JAMA 291:576-584

51. Erkan D, Vega JA, Ramon G, Kozora E, Lockshin MD (2013) A pilot open-label phase II trial of Rituximab for non-criteria manifestations of antiphospholipid syndrome. Arthr Rheum 65:464-475

52. Statkute L, Traynor A, Oyama Y et al (2005) Antiphospholipid syndrome in patients with systemic lupus erythematosus treated by autologous hematopoietic stem cell transplantation. Blood 106:2700-2709

53. Favaloro EJ, Wong RCW (2010) The antiphospholipid syndrome: a large elephant with many parts or an elusive chameleon disguised by many colours? Autoimmun Highlights 1:5-14
54. Espinosa G, Cervera R (2010) Management of the antiphospholipid syndrome. Autoimmun Highlights 1:15-22

55. Rai RS, Regan L, Clifford K et al (1995) Antiphospholipid antibodies and beta2-glycoprotein I in 500 women with recurrent miscarriage: result of a comprehensive screening approach. Hum Reprod 10:2001-2005

56. Rai RS, Clifford K, Cohen $\mathrm{H}$ et al (1995) High prospective fetal loss rate in untreated pregnancies of women with recurrent miscarriage and antiphospholipid antibodies. Hum Reprod 10:3301-3304

57. Galarza-Maldonado C, Kourilovitch MR, Perez-Fernandez O et al (2012) Obstetric antiphospholipid syndrome. Autoimmun Rev 11:288-295

58. Bates SM, Greer IA, Middeldorp S, Veenstra DL, Prabulos A-M, Vandvik PO (2012) VTE, thrombophilia, antithrombotic therapy, and pregnancy: antithrombotic therapy and prevention of thrombosis, 9th ed: American College of Chest Physicians evidence-based clinical practice guidelines. Chest 141(suppl):691S$736 \mathrm{~S}$

59. Bramham K, Hunt BJ, Germain S et al (2010) Pregnancy outcome in different clinical phenotypes of antiphospholipid syndrome. Lupus 19:58-64

60. Ruffatti A, Tonello M, Visentin S et al (2011) Risk factors for pregnancy failure in patients with anti-phospholipid syndrome treated with conventional therapies: a multicentre, case-control study. Rheumatology 50:1684-1689

61. Ruffatti A, Tonello M, Cavazzana A, Bagatella P, Pengo V (2009) Laboratory classification categories and pregnancy outcome in patients with primary antiphospholipid syndrome prescribed antithrombotic therapy. Thromb Res 123:482-487

62. Cervera R, Espinosa G (2012) Update on the catastrophic antiphospholipid syndrome and the "CAPS Registry". Semin Thromb Haemost 38:333-338

63. Cervera R (2012) CAPS registry. Lupus 21:755-757

64. Asherson RA, Cervera R, Piette JC et al (2001) Catastrophic antiphospholipid syndrome: clues to the pathogenesis from a series of 80 patients. Medicine (Baltimore) 80:355-376

65. Bucciarelli S, Espinosa G, Cervera R et al (2006) Mortality in the catastrophic antiphospholipid syndrome: causes of death and prognostic factors in a series of 250 patients. Arthr Rheum 54:2568-2576

66. Asherson RA, Cervera R, de Groot PR et al (2003) Catastrophic antiphospholipid syndrome: international consensus statement on classification criteria and treatment guidelines. Lupus 12: $530-534$

67. Erkan D, Asherson RA, Espinosa G et al (2003) Long term outcome of catastrophic antiphospholipid syndrome survivors. Ann Rheum Dis 62:530-533

68. Berman H, Rodriguez-Pintò I, Cervera R et al (2013) Rituximab use in the catastrophic antiphospholipid syndrome: descriptive analysis of the CAPS registry patients receiving rituximab. Autoimmun Rev 12:1085-1090

69. Broder A, Putterman C (2013) Hydroxychloroquine use is associated with lower odds of persistently positive antiphospholipid antibodies and/or lupus anticoagulant in systemic lupus erythematosus. J Rheumatol 40:30-33

70. Schmidt-Tanguy A, Voswinkel J, Henrion D et al (2013) Antithrombotic effects of hydroxychloroquine in primary antiphospholipid syndrome patients. J Thromb Haemost 11: 1927-1929

71. Lopez-Pedrera C, Ruiz-Limon P, Pedrera A et al (2011) Global effects of fluvastatin on the prothrombotic status of patients with antiphospholipid syndrome. Ann Rheum Dis 70:675-682 